\title{
A Modeling Framework to Investigate the Radial Component of the Pushrim Force in Manual Wheelchair Propulsion
}

\author{
Marko Ackermann ${ }^{1, a}$, Heitor Rogério Costa ${ }^{1}$ and Fabrizio Leonardi ${ }^{1}$ \\ ${ }^{1}$ FEI University, Mechanical Engineering Department, Av. Humberto de A. C. Castelo Branco, São Bernardo do Campo, Brazil
}

\begin{abstract}
The ratio of tangential to total pushrim force, the so-called Fraction Effective Force (FEF), has been used to evaluate wheelchair propulsion efficiency based on the fact that only the tangential component of the force on the pushrim contributes to actual wheelchair propulsion. Experimental studies, however, consistently show low FEF values and recent experimental as well as modelling investigations have conclusively shown that a more tangential pushrim force direction can lead to a decrease and not increase in propulsion efficiency. This study aims at quantifying the contributions of active, inertial and gravitational forces to the normal pushrim component. In order to achieve this goal, an inverse dynamics-based framework is proposed to estimate individual contributions to the pushrim forces using a model of the wheelchair-user system. The results show that the radial pushrim force component arise to a great extent due to purely mechanical effects, including inertial and gravitational forces. These results corroborate previous findings according to which radial pushrim force components are not necessarily a result of inefficient propulsion strategies or hand-rim friction requirements. This study proposes a novel framework to quantify the individual contributions of active, inertial and gravitational forces to pushrim forces during wheelchair propulsion.
\end{abstract}

\section{Introduction}

In spite of the large number of users worldwide, locomotion with manual wheelchairs is associated with upper-extremity injuries, shoulder pain, large energy demand and low efficiency [1-3]. The inefficiency of wheelchair locomotion has been linked to the radial component of the push force during the propulsion phase $[4,5]$, based on the fact that only the tangential component of the force on the rim contributes to the moment applied to wheel and, therefore, to actual wheelchair propulsion. According to this reasoning, radial forces arise exclusively as a result of suboptimal propulsion strategies and pushrim friction requirements.

However, many experimental studies have shown low ratios of tangential force to total pushrim force, the so-called Fraction Effective Force (FEF), during wheelchair propulsion even for experienced users [6,7]. This indicates large FEF with a more tangential pushrim force direction is not necessarily associated with higher efficiency. In fact, de Groot and colleagues [8] show that maximization of FEF by instructing subjects to direct pusrim forces tangentially did not lead to an increase in propulsion efficiency and Bregman and colleagues [9] show that a tangential force condition led to a $30 \%$ higher physiological cost. In the latter study authors conclude that the observed force direction, with relatively large radial component in wheelchair propulsion, is a compromise between efficiency and the constraints imposed by the wheelchair-user system.

Therefore, while it is true that only the tangential pushrim force contributes to mechanical work, radial forces arise as a result of musculoskeletal structure and mechanical constraints imposed by the wheelchair-user system. A simple example is the effect of the upper extremities weight, which is partially supported by the pushrim, in the absence of upper-extremity muscular activity. In this case, radial forces arise and are clearly not linked to inefficiencies. Attempting to eliminate the radial component would require muscle activation and, consequently, greater energy consumption.

Nevertheless, other mechanical, physiological and musculoskeletal influences on radial pushrim forces are less obvious and are still not well understood. In particular, the role of the inertial forces has not been addressed in the literature to the best of our knowledge despite the large accelerations undergone by the upper extremities, even at moderate locomotion speeds. Quantifying these individual contributions requires a model of the wheelchair-user system, but the models proposed in the literature so far [10-14] have not been employed to address this particular question.

In this context, the primary aim of this study is investigating the contributions of active, gravitational and inertial forces to the radial pushrim force during the propulsion phase of manual wheelchair locomotion. In order to achieve this goal, we adopt a mechanical model

\footnotetext{
a Corresponding author: mackermann@fei.edu.br
} 
of the wheelchair-user system and propose an inverse dynamics-based framework.

\section{Methods}

\subsection{Model}

We develop a planar wheelchair-user system model composed of four rigid bodies assuming bilateral symmetry: both wheels, both upper arms, both forearms, and the remaining segments along with the chair lumped in a single rigid body, Fig. 1 . The shoulder and the elbow are modeled as ideal hinge joints driven by active moments, $\tau_{s}$ and $\tau_{e}$, respectively. It is assumed that there is no slip between wheels and ground and that the shoulder joint does not move relative to the wheelchair, as usual in models of wheelchair locomotion [10-14].

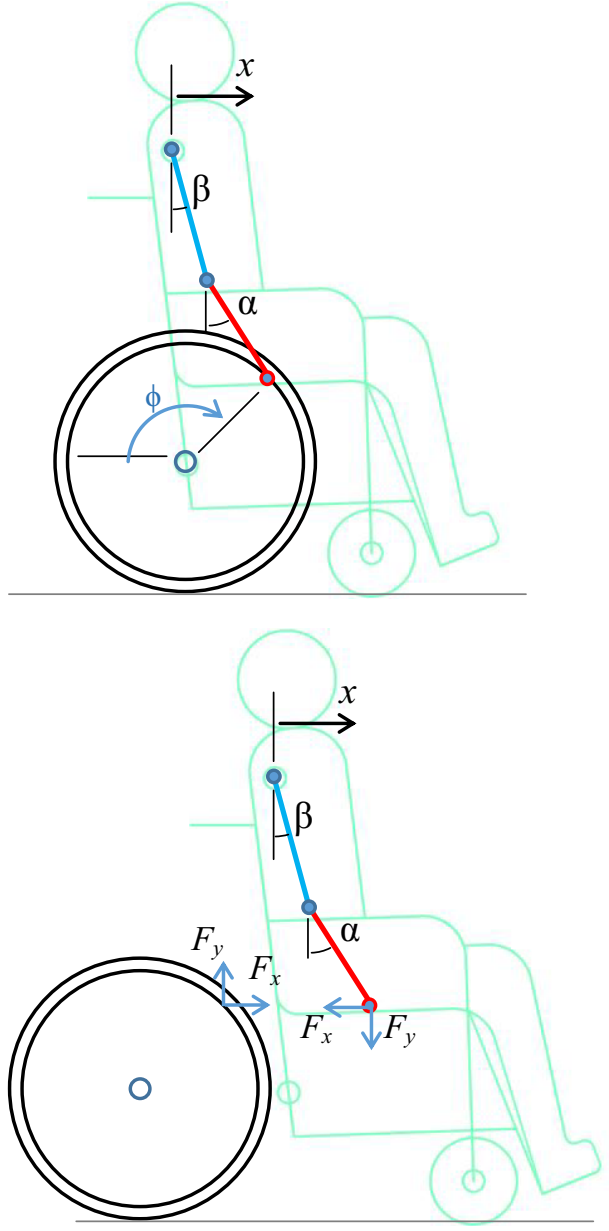

Figure 1. Model of the wheelchair-user system in the propulsion phase: wheel (black), upper arm (blue), forearm (red) and remaining body segments and wheelchair (green). On the right, the contact forces between hands and pushrim are depicted.

The mass, moments of inertia, center of mass locations and segment lengths are estimated using anthropometric data from [15] and from the software OpenSim [16,17] for a $1.7 \mathrm{~m}, 70 \mathrm{~kg}$ person. The wheelchair dimensions and inertia properties are estimated from data of a commercially available manual wheelchair. All adopted values are reported in Table 1 of the Appendix. The rolling resistance $F_{\text {roll }}$ is modeled as a constant horizontal force applied to the wheels. We adopted a total value of 30 $\mathrm{N}$ for both wheels which is consistent with values reported in [3].

The adopted generalized coordinates $q$, are the angle between the upper arm and the vertical, $\beta$, the angle between the forearm and the vertical, $\alpha$, and the horizontal displacement of the wheelchair and shoulder joint, $x$, as $q=\left[\begin{array}{lll}x & \beta & \alpha\end{array}\right]^{T}$. In the propulsion phase, as in Fig. 1, the hands are in contact with the pushrims. This interaction is modeled as an ideal hinge joint forming a closed-loop kinematic chain with a single degree of freedom that can be interpreted as a moving four-bar mechanism. The hand-rim contact imposes two kinematic constraints, shown in details in the Appendix, as

$$
c(x, \beta, \alpha)=0 .
$$

The equations of motion of the wheelchair-user system depicted in Fig. 1 were derived using the Newton-Euler Formalism [18] incorporating the hand-rim contact forces into de equations of motion as

$$
\begin{aligned}
& M(q) \ddot{q}+k(q, \dot{q})=k_{G}(q)+G(q)\left[\begin{array}{l}
F_{x} \\
F_{y}
\end{array}\right]+ \\
& H(q)\left[\begin{array}{l}
\tau_{s} \\
\tau_{e}
\end{array}\right]+Q\left[F_{\text {roll }}\right],
\end{aligned}
$$

where $M$ is the mass matrix, $k$ is the vector of generalized Coriolis and centrifugal forces, $k_{G}$ is the vector of generalized forces due to gravity, $G$ transforms the horizontal $F_{x}$ and vertical $F_{y}$ components of the pushrim force (Fig. 1) in generalized forces, $H$ transforms the shoulder moment $\tau_{s}$ and the elbow moment $\tau_{e}$ in generalized forces, and $Q$ transforms the rolling resistance force $F_{\text {roll }}$ in generalized force. All the matrices in Eq. (2) are presented in Tab. 2 in the Appendix.

$$
F_{y}
$$

\subsection{Inverse Kinematics}

The kinematics of the multibody system, $q(t), \dot{q}(t)$ and $\ddot{q}(t)$, is reconstructed from the motion profile of the wheelchair, $x(t), \dot{x}(t)$ and $\ddot{x}(t)$, using the constraint equations, Eq. (1), and its first and second time derivatives,

$$
\begin{aligned}
& c_{q} \dot{q}=0, \\
& c_{q} \ddot{q}+\dot{c}_{q} \dot{q}=0,
\end{aligned}
$$

to determine $\beta(t), \alpha(t), \dot{\beta}(t), \dot{\alpha}(t), \ddot{\beta}(t)$ and $\ddot{\alpha}(t)$ for each considered time instant, where $c_{q}=\partial c / \partial q$ is the Jacobian of the constraints equation. In this study, a constant wheelchair speed $\dot{x}=v$ is imposed, with $\ddot{x}=0$.

\subsection{Estimation of Joint Moments and Pushrim Forces}

After the kinematics of the multibody system is reconstructed, it is possible to estimate the joint moments, $\tau_{s}$ and $\tau_{e}$, and the associated pushrim contact forces, $F_{x}$ and $F_{y}$, for given values of the rolling resistance force $F_{\text {roll }}$ and of the wheelchair speed $v$, from Eq. (2) as 
$\left[\begin{array}{ll}G(q) & H(q)\end{array}\right]\left[\begin{array}{l}F_{x} \\ F_{y} \\ \tau_{s} \\ \tau_{e}\end{array}\right]=M(q) \ddot{q}+k(q, \dot{q})-k_{G}(q)-$ $Q\left[F_{\text {roll }}\right]$

Note that there are three equations of motion in Eq. (5) and four unknowns $\left(\tau_{s}, \tau_{e}, F_{x}, F_{y}\right)$ leading to an indeterminate problem. This means that there are infinite possible solutions for the joint moments that generate the same motion pattern. This problem is solved by assuming the central nervous system selects joint moments such as to minimize an appropriate cost function representing, for instance, energy consumption or "effort" [19]. In this study, we adopt as cost function the sum of the squared joint moments as

$$
J=\tau_{s}^{2}+\tau_{e}^{2}
$$

which is consistent with cost functions frequently adopted to solve the force-sharing problem in biomechanics [19]. Thus, an optimization problem is formulated as: find the joint moments, $\tau_{s}$ and $\tau_{e}$, and the pushrim force components, $F_{x}$ and $F_{y}$, that minimize the cost function in Eq. (6) and satisfy the equations of motion in Eq. (5). This problem is solved using the quadprog function of Matlab, which implements a quadratic programming solver.

In order to compare the results obtained by the aforementioned optimization with the corresponding results for a condition in which a perfectly tangent total pushrim force is imposed, we add a constraint on $F_{x}$ anf $F_{y}$ that imposes zero radial force, $F_{r}=0$, and solve the resulting set of algebraic equations for $\tau_{s}, \tau_{e}, F_{x}$ and $F_{y}$.

\subsection{Contribution of gravitational, inertial and active forces}

In order to estimate the individual contributions of the inertial, gravitational and active forces to the pushrim force, we decompose Eq. (2) into three sets of equations, each accounting for one of these contributions, as

$$
\begin{aligned}
& 0=G(q)\left[\begin{array}{l}
F_{x, a} \\
F_{y, a}
\end{array}\right]+H(q)\left[\begin{array}{l}
\tau_{s} \\
\tau_{e}
\end{array}\right]+Q\left[F_{\text {ext }, a}\right], \\
& M(q) \ddot{q}+k(q, \dot{q})=G(q)\left[\begin{array}{l}
F_{x, i} \\
F_{y, i}
\end{array}\right]+Q\left[F_{\text {ext }, i}\right], \\
& 0=k_{G}(q)+G(q)\left[\begin{array}{l}
F_{x, g} \\
F_{y, g}
\end{array}\right]+Q\left[F_{\text {ext }, g}\right],
\end{aligned}
$$

Note that the sum of Eqs. (7), (8) and (9) yields Eq. (2) for

$\left[\begin{array}{l}F_{x} \\ F_{y}\end{array}\right]=\left[\begin{array}{l}F_{x, a} \\ F_{y, a}\end{array}\right]+\left[\begin{array}{l}F_{x, i} \\ F_{y, i}\end{array}\right]+\left[\begin{array}{l}F_{x, g} \\ F_{y, g}\end{array}\right]$, and

$$
F_{\text {roll }}=F_{\text {ext }, a}+F_{\text {ext }, i}+F_{\text {ext }, g} .
$$

Solving Eq. (7) for $F_{x, a}, F_{y, a}$ and $F_{\text {ext,a }}$ from the previously computed joint moments gives the pushrim force components $F_{x, a}$ and $F_{y, a}$ due to the active joint moments only, i.e. for null inertial $(M(q) \ddot{q}+k(q, \dot{q})=0)$ and gravitational $\left(k_{G}=0\right)$ terms. The computed $F_{\text {ext,a }}$ is the external force, as a fraction of $F_{\text {roll }}$, necessary to keep the constant velocity of the wheelchair and maintain the static equilibrium.

Solving Eq. (8) for $F_{x, i}, F_{y, i}$ and $F_{\text {ext, } i}$ gives the pushrim force components, $F_{x, i}$ and $F_{y, i}$, due to the inertial terms only, i.e. for null active moments $\left(\tau_{s}=\tau_{e}=0\right)$ and gravitational forces $\left(k_{G}=0\right)$. These would be the forces arising on the pushrim if a constant velocity is imposed to the wheelchair, if user does not activate muscles and if segment weights are set to zero. The computed $F_{\text {ext }, i}$ is the external force, as a fraction of $F_{\text {roll }}$, necessary to keep the constant velocity of the wheelchair. Note that the pushrim force due to inertial effects do not depend on the joint moments.

Solving Eq. (9) for $F_{x, g}, F_{y, g}$ and $F_{\text {ext,g }}$ gives the pushrim force components, $F_{x, g}$ and $F_{y, g}$, due to the gravitational terms only, i.e. for null active moments $\left(\tau_{s}=\tau_{e}=0\right)$ and inertial terms $(M(q) \ddot{q}+k(q, \dot{q})=0)$. These would be the forces arising on the pushrim in a quasi-static condition, i.e. for a low wheelchair speed. The computed $F_{\text {ext, }}$ is the external force, as a fraction of $F_{\text {roll }}$, necessary to guarantee static equilibrium in this condition. Note that these pushrim contact forces do not depend on the joint moments.

Therefore, the total pushrim force computed by solving Eq. (5) is the sum of the active, inertial and gravitational contributions, obtained by solving Eqs. (7), (8) and (9), respectively, as in Eq. (10).

\section{Results}

The first analysis involved the estimation of joint moments and pushrim forces for a prescribed wheelchair velocity $v$ by using the developed model and the proposed optimization framework. Fig. 2 depicts a stick-figure representation of the system kinematics and the estimated pushrim force at a constant wheelchair speed of $1.0 \mathrm{~m} / \mathrm{s}$. Fig. 3 shows other relevant profiles. The Fraction Effective Force (FEF), defined in [6] as the ratio of the pushrim tangent component to the pushrim total force is shown on the left-hand side of Fig. 3. The individual contributions of the active, inertial and gravitational terms on the equations of motion to the total radial pushrim force are shown on the right-hand side of Fig. 3.

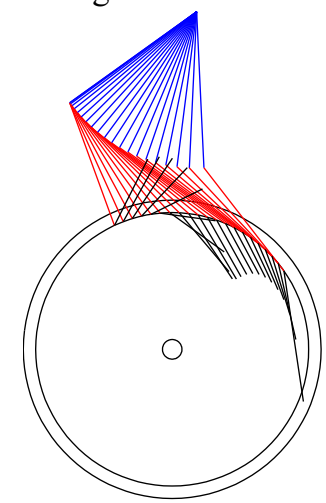

Figure 2. Stick figures of the reconstructed system kinematics and the estimated pushrim forces, with direction and magnitude represented by the black segments, for a wheelchair velocity $\mathrm{v}=$ $1.0 \mathrm{~m} / \mathrm{s}$. 

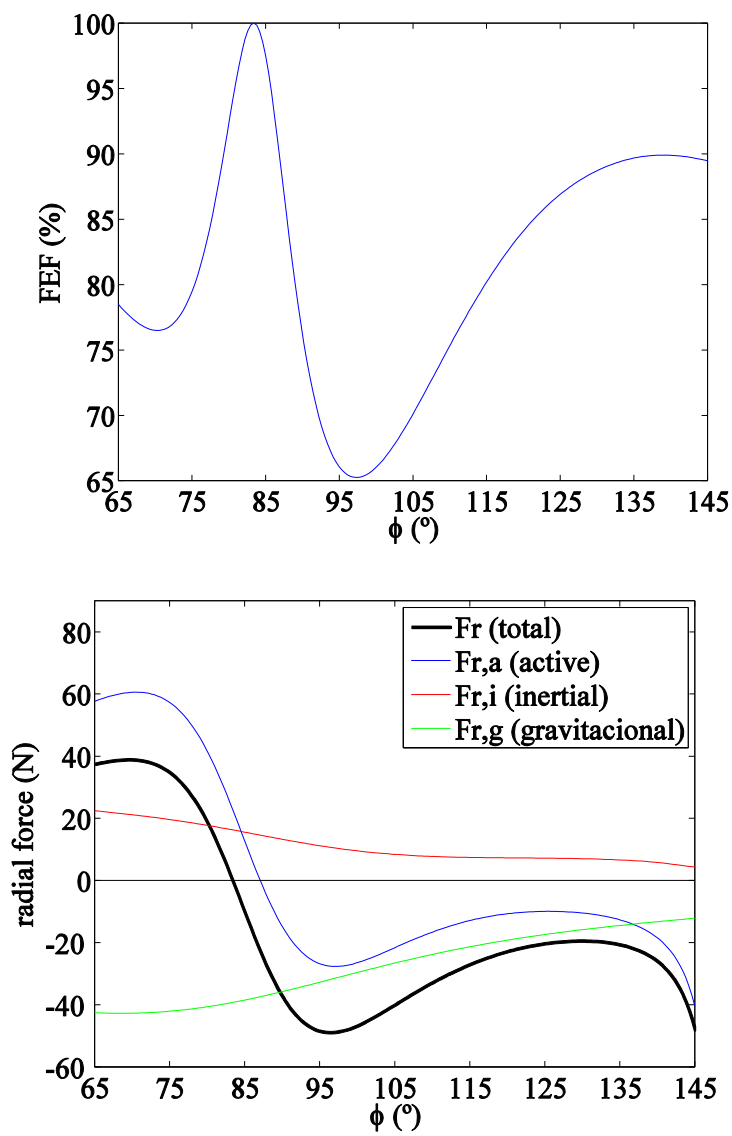

Figure 3. Estimated Fraction Effective Force (FEF), on the left, and individual pushrim radial force contributions, on the right, for each rear wheel angular position $\phi$ along the propulsion phase for a wheelchair velocity $\mathrm{v}=1.0 \mathrm{~m} / \mathrm{s}$.

The second analysis involved numerically determining the joint moments and pushrim forces by imposing a purely tangent pushrim force, i.e. by imposing a zero radial force, $F_{r}=0$. This resulted in different joint moment profiles compared to the joint moment profiles determined in the first analysis, as shown on the left-hand side of Fig. 4. The right-hand side of Fig. 4 shows the estimated contribution of the inertial forces to the radial pushrim component at different wheelchair velocities.

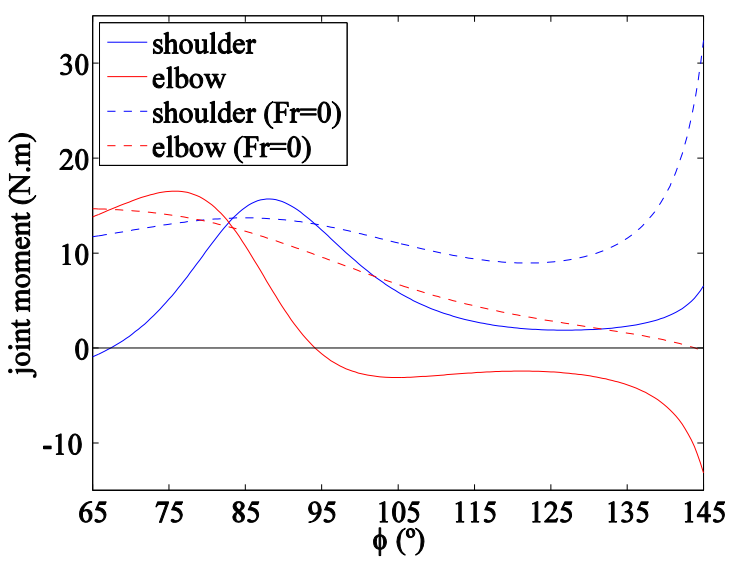

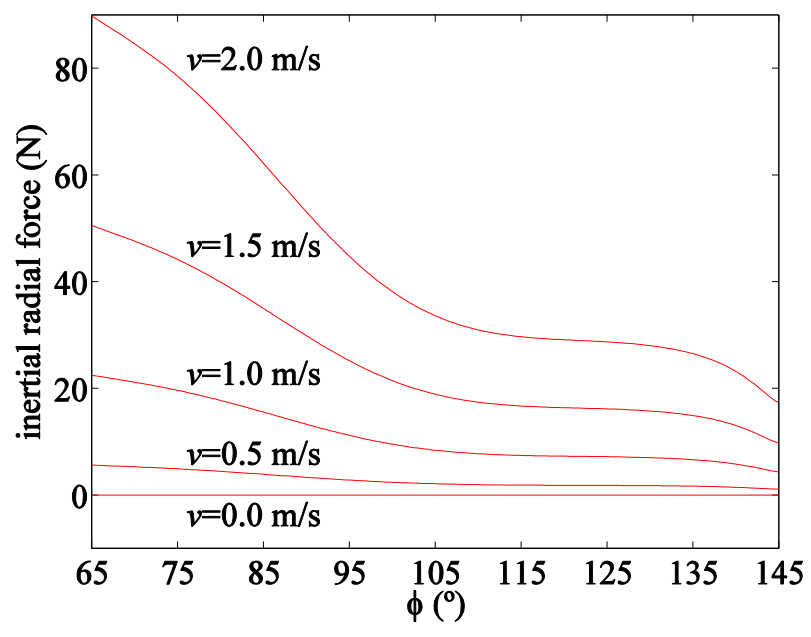

Figure 4. Left: joint moments estimated by solving the optimization problem compared with the joint moments obtained by imposing $F_{r}=0$, for a wheelchair velocity $v=1.0 \mathrm{~m} / \mathrm{s}$. Right: estimated contribution of inertial forces to the pushrim radial force for different wheelchair velocities.

\section{Discussion and Conclusion}

A modeling and optimization framework was proposed to estimate pushrim forces and upper limb joint moments from the wheelchair motion profile using a mechanical model of the wheelchair-user system during the propulsion phase. The proposed framework was employed to investigate the individual contributions of gravitational and inertial forces as well as of shoulder and elbow active moments to the radial component of the pushrim force.

The results corroborate more recent findings on the inadequacy of using the FEF as a measure of propulsion efficiency [8,9]. The estimated FEF values, Fig. 3 (left), are consistent with data published in the literature for the investigated velocity, although the average value of $81 \%$ estimated here is slightly greater than reported average values, ranging from 68 to $77 \%$ [3]. Note that this fact evidences that the low FEF values observed are probably not only linked to physiological aspects but also and importantly to purely mechanical aspects of the multibody system. In fact, as shown on the right-hand side of Fig. 3, the gravitational and inertial contributions have the same order of magnitude as the active contribution due to the shoulder and elbow actuation.

Conversely, it is important to observe that even neglecting the inertial and gravitational effects and isolating the effect of the shoulder and elbow joint moments, large radial pushrim force components arise. If we recall that these results are obtained by minimizing joint moments, it gets evident that the radial force is a natural consequence of the multibody system topology and not necessarily a byproduct of inefficient propulsion strategies. The results shown on the left-hand side of Fig. 4 corroborate this conclusion. In this figure, the joint moments obtained through the optimization approach are compared to the joint moments obtained by imposing a null radial force component, or a perfectly tangent 
pushrim force. It is possible to observe that the joint moments in the latter case (for $F_{r}=0$ ) are larger than the optimal moments for almost the whole angular position span, indicating that imposing a tangent total pushrim force will lead to greater and not lower propulsion effort, a finding already shown conclusively by [9].

It is interesting to note that the gravitational effect is larger than the inertial effect at the speed of $1.0 \mathrm{~m} / \mathrm{s}$ and that they contribute to the radial component in opposite directions. While segment weights give rise to radial forces on the pushrim directed inwards to the center of the wheel, the radial force due to inertial forces points outwards. The gravitational effect will not change with wheelchair speed in opposition to the effect of the inertial forces, which is greatly influenced by the wheelchair speed as shown on the right-hand side of Fig. 4. Note that the inertial effect on the radial pushrim component becomes greater than the gravitational effect for wheelchair velocities greater than $1.5 \mathrm{~m} / \mathrm{s}$, a moderate locomotion speed.

The presented results shed some light on the individual contributions of active, inertial and gravitational forces and evidence that radial pushrim components are to a great extent a result of purely mechanical aspects, including the nature of the multibody system kinematic chain, segments' weight and inertial forces. This evidences that the appearance of a radial force component on the pushrim is not necessarily due to suboptimal propulsion strategies or friction requirements, as suggested by some authors. As already pointed out in other studies, the FEF values are consistently low in wheelchair propulsion and instructing a more tangential pushrim force can lead to an increase in actuation effort and a decrease in propulsion efficiency.

The imposition of a constant wheelchair speed can be mentioned as a limitation of this study, as in reality the speed fluctuates around the average speed over the propulsion phase. However, we believe this does not invalidate the conclusions drawn. In an ongoing study, the proposed framework will be applied to measured speed profiles obtained by means of a tachometer connected to the rear wheel of the wheelchair. This same approach can be extended to cases in which more information on system kinematics is available or in a predictive optimal control framework $[10,11,14,20]$.

\section{Acknowledgements}

We gratefully acknowledge the support of the Brazilian Ministry of Science, Technology and Innovation (MCTI) and of the National Council for Scientific and Technological Development (CNPq) through the grant 458717/2013-4.

\section{References}

1. R.A. Cooper, L.A. Quatrano, P.W. Axelson, W. Harlan, Research on physical activity and health among people with disabilities: a consensus statement, Journal of Rehabilitation Research and Development. 36(2) (1999) 142.
2. M.L. Boninger, A.L. Souza, R.A. Cooper, S. G. Fitzgerald, A.M. Koontz, B.T. Fay, Propulsion patterns and pushrim biomechanics in manual wheelchair propulsion, Arch Phys Med Rehabil. 83 (2002) 718-723.

3. L.H.V. van der Woude, H.E.J. Veeger, A.J. Dallmeijer, T.W.J. Janssen, L.A. Rozendaal, Biomechanics and physiology in active manual wheelchair propulsion, Med Eng Phys. 23 (2001) 713-733.

4. H.W. Wu, L.J. Berglund, F.C. Su, B. Yu, A. Westreich, K.J. Kim, K.N. An, An instrumented wheel for kinetic analysis of wheelchair propulsion, Journal of Biomechanical Engineering. 120(4) (1998) 533-535.

5. M.L. Boninger, R.A. Cooper, R.N. Robertson, S.D. Shimada, Three-Dimensional pushrim forces during two speeds of wheelchair propulsion, American Journal of Physical Medicine \& Rehabilitation. 76(5) (1997), 420-426.

6. H.E.J. Veeger, L.H.V. van der Woude, Load on the upper extremity in manual wheelchair propulsion, Journal of Electromyography and Kinesiology. 1 (1991) 270-280.

7. B.R. Kotajarvi, B.S. Michelle, An Kai-Nan, D.Z. Kristin, R.K. Kenton, R.B. Jeffrey, The effect of seat position on wheelchair propulsion biomechanics, Journal of Rehabilitation Research and Development. 41(3B) (2004) 403-414.

8. S. de Groot, H.E.J. Veeger, P. Hollander, L.H.V. van der Woude, Wheelchair propulsion technique and mechanical efficiency after 3 wk of practice, Medicine \& Science in Sports \& Exercise. 34 (2002) 756-766.

9. D.J.J. Bregman, S. van Drongelen, H.E.J. Veeger, Is effective force application in handrim wheelchair propulsion also efficient?, Clinical Biomechanics. 24 (2009) 13-19.

10. J.W. Rankin, A. M. Kwarciak, W. M. Richter, R. R. Neptune, The influence of altering push force effectiveness on upper extremity demand during wheelchair propulsion, Journal of Biomechanics. 43 (2010) 2771-2779.

11. J. W. Rankin, W. M. Richter, R. R. Neptune, Individual muscle contributions to push and recovery subtasks during wheelchair propulsion, Journal of Biomechanics. 44 (2011) 1246-1252.

12. J.W. Rankin, A. M. Kwarciak, W. M. Richter, R. R. Neptune, The influence of wheelchair propulsion technique on upper extremity muscle demand: a simulation study. Clinical Biomechanics. 27(9), 879-886.

13. M. Leary, J. Gruijters, M. Mazur, A. Subic, M. Burton, F. K. Fuss, A fundamental model of quasi-static wheelchair biomechanics, Medical Engineering and Physics. 34 (2012) 1278-1286.

14. M. Ackermann, F. Leonardi, H.R. Costa, A.T. Fleury, Modeling and optimal control formulation for manual wheelchair locomotion: The influence of mass and slope on performance, Proc. of The 5th IEEE RAS \& EMBS International Conference on Biomedical Robotics and Biomechatronics. (2014) 1079-1084. 
15. D. A. Winter, Biomechanics and Motor Control of Human Movement, John-Wiley \& Sons Inc., New York, 2009, 4th ed.

16. K.R.S. Holzbaur, W.M. Murray, S.L. Delp, A model of the upper extremity for simulating musculoskeletal surgery and analyzing neuromuscular control, Annals of Biomedical Engineering. 33(6) (2005) 829-840.

17. S.L. Delp, C.A. Frank, S.A. Allison, P. Loan, A. Habib, C.T. John, E. Guendelman, D.G. Thelen, OpenSim: open-source software to create and analyze dynamic simulations of movement, IEEE
Transactions on Biomedical Engineering. 54(11) (2007) 1940-1950.

18. W. Schiehlen, Multibody system dynamics: roots and perspectives, Multibody System Dynamics. 1 (1997) 149-188.

19. A. Erdemir, S. McLean, W. Herzog, A. J. van den Bogert, Model-based estimation of muscle forces exerted during movements, Clinical Biomechanics. 22 (2007) 131-154.

20. M. Ackermann, A. J. van den Bogert, Optimality principles for model-based prediction of human gait, Journal of Biomechanics. 43 (2010) 1055-1060. 


\section{Appendix}

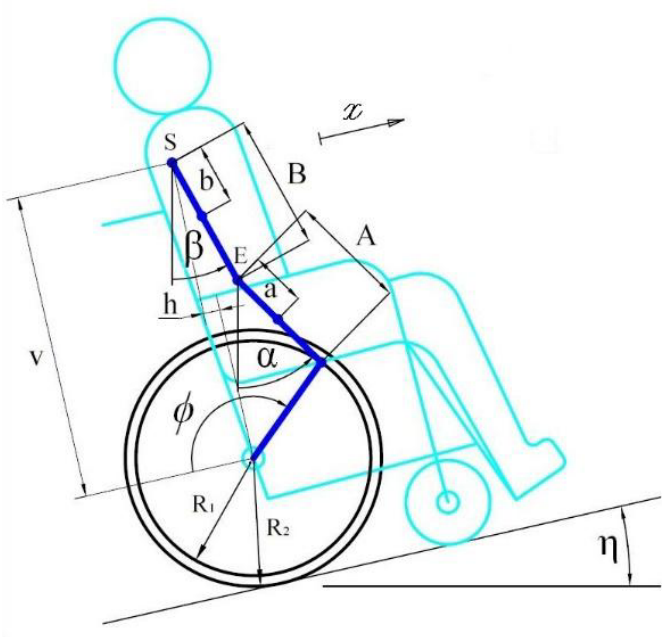

Table 1 Anthropometric and Wheelchair Data

\begin{tabular}{|c|c|}
\hline Person Height & $1.70 \mathrm{~m}$ \\
\hline Person mass & $70.0 \mathrm{~kg}$ \\
\hline $\begin{array}{l}\text { Shoulder to axle distance - } \\
\text { horizontal (h) }\end{array}$ & $0.05 \mathrm{~m}$ \\
\hline $\begin{array}{l}\text { Shoulder to axle distance }- \text { vertical } \\
\text { (v) }\end{array}$ & $0.69 \mathrm{~m}$ \\
\hline Upper arm length (B) & $0.3196 \mathrm{~m}$ \\
\hline Upper arm CM location (b) & $0.1995 \mathrm{~m}$ \\
\hline Forearm length (A) & $0.2667 \mathrm{~m}$ \\
\hline Forearm + hand CM location (a) & $0.2006 \mathrm{~m}$ \\
\hline Handrim radius $\left(\mathrm{R}_{1}\right)$ & $0.2794 \mathrm{~m}$ \\
\hline Rear wheel radius $\left(\mathrm{R}_{2}\right)$ & $0.3048 \mathrm{~m}$ \\
\hline $\begin{array}{l}\text { Moment of inertia of both rear } \\
\text { wheels }\left(\mathrm{J}_{\mathrm{R}}\right)\end{array}$ & $\begin{array}{l}0.3716 \\
\mathrm{~kg} \cdot \mathrm{m}^{2}\end{array}$ \\
\hline $\begin{array}{l}\text { Moment of inertia of both upper } \\
\operatorname{arms}\left(\mathrm{J}_{\mathrm{b}}\right)\end{array}$ & $\begin{array}{l}0.0333 \\
\mathrm{~kg} . \mathrm{m}^{2}\end{array}$ \\
\hline $\begin{array}{l}\text { Moment of inertia both forearms }+ \\
\text { hands }\left(\mathrm{J}_{\mathrm{a}}\right)\end{array}$ & $\begin{array}{l}0.0453 \\
\mathrm{~kg} . \mathrm{m}^{2}\end{array}$ \\
\hline Mass of both forearms + hands $\left(\mathrm{m}_{\mathrm{A}}\right)$ & $2.84 \mathrm{~kg}$ \\
\hline Mass of both upper arms $\left(\mathrm{m}_{\mathrm{B}}\right)$ & $3.45 \mathrm{~kg}$ \\
\hline Mass of both wheels $\left(\mathrm{m}_{\mathrm{R}}\right)$ & $4.00 \mathrm{~kg}$ \\
\hline $\begin{array}{l}\text { Total mass excluding arms and } \\
\text { wheels }\left(\mathrm{m}_{\mathrm{C}}\right)\end{array}$ & $75.714 \mathrm{~kg}$ \\
\hline Gravity acceleration $(\mathrm{g})$ & $9.81 \mathrm{~m} / \mathrm{s}^{2}$ \\
\hline $\begin{array}{l}\text { Total rear wheels rolling resistance } \\
\left(\mathrm{F}_{\text {roll }}\right)\end{array}$ & $-30 \mathrm{~N}$ \\
\hline
\end{tabular}

Table 2 Matrices of Eq. (2)

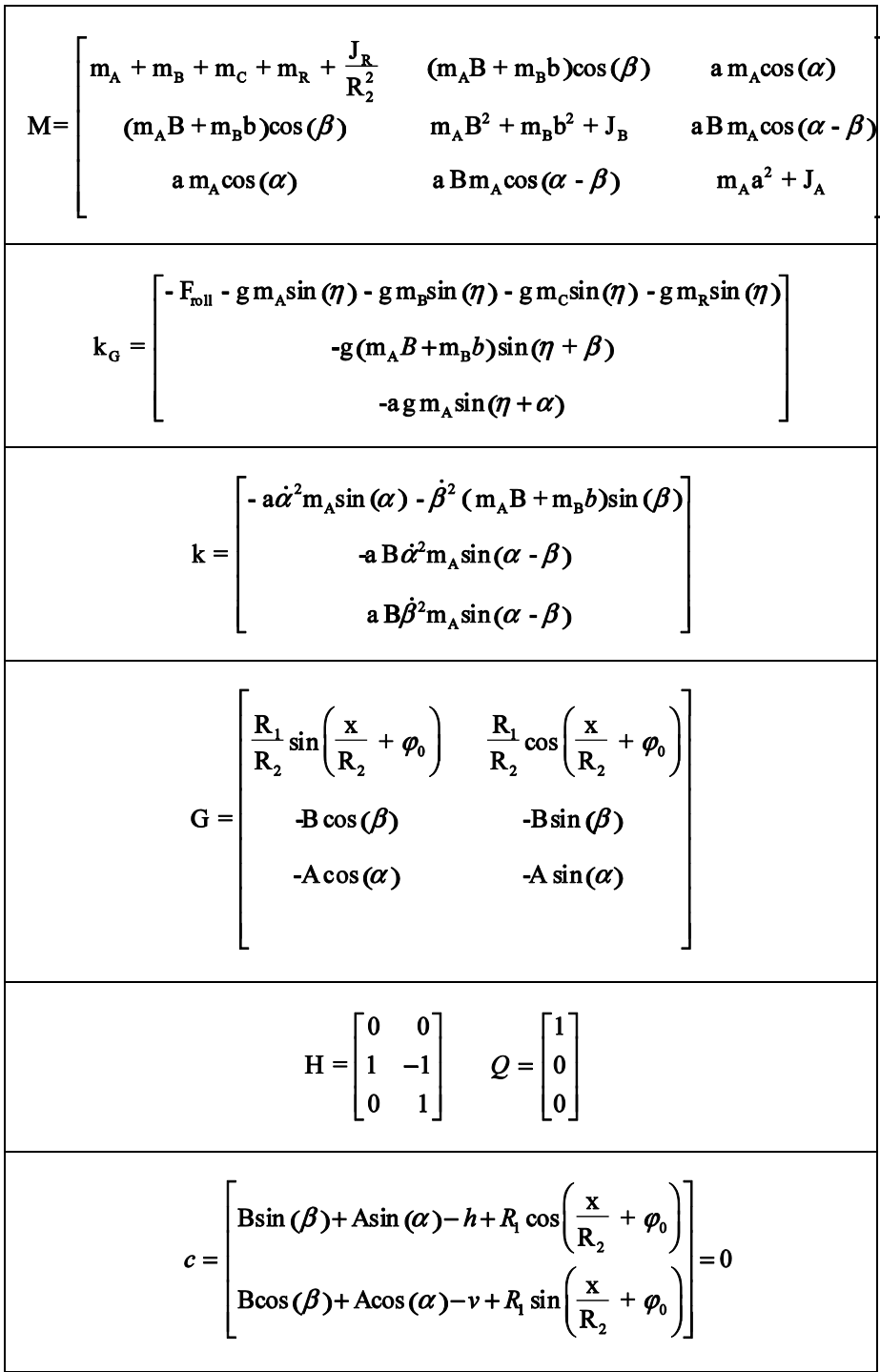


MATEC Web of Conferences

\begin{tabular}{l|l}
\hline Slope angle $(\eta)$ & $0^{\circ}$ \\
\hline Initial angular position $\left(\phi_{0}\right)$ & $65^{\circ}$ \\
\hline Final angular position $\left(\phi_{\mathrm{f}}\right)$ & $145^{\circ}$ \\
\hline
\end{tabular}

